\title{
Percepción de la calidad de servicio del usuario interno en una institución pública
}

Suseyla Roxana Sarmiento Prieto ${ }^{1}$ Mildred Teresa Paredes Tarazona ${ }^{2}$

\begin{abstract}
RESUMEN
Este es un estudio cuantitativo, descriptivo y comparativo sobre una muestra de 310 servidores públicos. Tiene por objetivo describir la percepción de la calidad de servicio del usuario interno mediante los factores de cooperación entre usuarios internos, liderazgo de la gestión, responsabilidad social institucional y valoración de su labor. Para ello, se parte de la consideración del usuario interno como aquel colaborador que se desempeña en cualquier posición de la estructura organizacional. Asimismo, se aplicó la escala ordinal de cinco grados EPCSU-IP válida y confiable para la investigación. La percepción total es a nivel neutro $(3,54)$ con tendencia a grados mayores; tiende a ser favorable en las dimensiones de cooperación entre usuarios internos $(3,67)$ y valoración de su labor $(3,73)$, y desfavorable en los factores liderazgo de la gestión $(3,45)$ y responsabilidad social institucional $(3,27)$. Esta variable es estudiada como indicador del clima organizacional en lo referente a la gestión de los procesos productivos de bienes o servicios.
\end{abstract}

Palabras-claves: Calidad de servicio; usuario interno; gestión; responsabilidad social institucional; clima organizacional.

\section{INTRODUCCIÓN}

Las instituciones públicas en el Perú, como organizaciones burocráticas, prestan y centralizan su interés en el cumplimiento de la norma y la fiscalización de la actividad realizada (Lapassade, 1977). A través de los años, por la naturaleza de la construcción social de estas organizaciones (Miguel, 1999), se ha descuidado la calidad de servicio que brindan al usuario externo, causando malestar a la población, la cual califica al servicio como lento, malo, burocrático, corrupto e incompetente. Este descontento se expresa en el incremento de quejas por parte del cliente externo. Por otro lado, hay también una falta de interés por parte del cliente interno en brindar un buen servicio.

La mayor producción y esfuerzos se centran en la satisfacción del usuario externo o cliente, con el propósito de lograr, entre otras razones, su fidelidad; sin embargo, el usuario interno o cliente interno es ignorado (Balmori y Flores, 2014). Por esta razón, la gerencia que no propicia una gestión sobre lo anterior tiende a afectar los procesos institucionales, debido a que los usuarios internos no son conscientes de que prestan un servicio a sus compañeros, impactando de forma favorable y eficiente en su trabajo. Esto conduce a que el trabajador olvide que es un proveedor interno de sus demás compañeros y de otros órganos de la institución.

Los usuarios internos son considerados como aliados estratégicos dentro de las organizaciones e instituciones sin fines de lucro, pues son el soporte del grupo, incrementan las ganancias y posicionan el negocio gracias a sus productos y servicios (Domínguez, 2006). Además, son el artificio en el logro de los objetivos estratégicos, puesto que "ofrecerán no solo un servicio sino una calidad de servicio que logre sobrepasar las expectativas del cliente externo, llamar su atención, lograr esa fidelización que la empresa necesita y despertar el deseo del cliente de volver" (Simancas, 2012, p. 89).

Por ello, es importante entender que cuando los usuarios internos realizan sus funciones interactúan de diversa manera,

\footnotetext{
Psicóloga, Gerente general de Sugrafica en Inversiones Sujuro S. A. C., Lima, Perú. E-mail: ssarmiento@sugrafica.com

2 Dra. en Psicología, docente principal de la Facultad de Psicología de la Universidad Nacional Mayor de San Marcos. Lima, Perú E-mail: mparedest@unmsm.edu.pe
} 
ello se evidencia mediante sus comportamientos individuales y grupales que, por lo general, no están encaminados a brindar un buen servicio al cliente externo. Asimismo, se expresa en la contraposición de organizaciones que promueven un ambiente basado en el clima de servicio positivo que fortalece el comportamiento del trabajador al buen servicio de los clientes (Schneider, White y Paul, 1998). Esta variabilidad en la actitud de los trabajadores está relacionada con la fortaleza del clima; y, esta última, con las experiencias de los clientes (Schneider y Nicole, 2002), hecho que se manifiesta en una personalidad madura (Argyris, 1973). La calidad del servicio interno influye positivamente en los comportamientos prosociales por el rol y la cooperación entre trabajadores, lo que lleva a concluir que la prestación de servicios internos de alta calidad aumenta o mantiene la satisfacción de los clientes (Bellou y Andronikidis, 2008). Estos comportamientos prosociales son la razón para gestionar la responsabilidad social institucional, ya que al jugar un papel importante en el desarrollo personal del usuario interno, a través del servicio valorado en la satisfacción del cliente, se logra más que una simple práctica de marketing comercial.

El usuario interno, agente de cambio y promotor y actor de la producción, requiere tener las condiciones necesarias para desempeñarse motivado y con óptimo potencial, alineando sus expectativas con los objetivos estratégicos de la institución. Esto es expresado en la conducta de cooperación y de participación entre usuarios internos, comportamientos organizacionales que potencializan la capacidad de resolver y ofrecer servicios colectivos en base a un sentimiento de pertenencia a una comunidad (Adler, 1973).

La percepción de la calidad de servicio es "el resultado de un proceso de evaluación, donde el cliente compara sus expectativas con el servicio que percibe que ha recibido [...] resultado de este proceso será la calidad percibida del servicio" (Grönroos 1984, p. 37) [traducción nuestra]. Por esta razón, cuando el cliente recibe un servicio emite un juicio de valor en base a las expectativas que tenía en un principio (Balmori y Flores, 2014). La satisfacción del usuario interno se produce en base a todo un procedimiento, dado que el consumidor (usuario interno) no solo está interesado en el producto o servicio, sino en todo el proceso (Grönroos, 1984), lo cual involucra a otros factores que le generen una experiencia agradable frente a la entrega de servicio. Schneider, White y Paul (1998) plantean el siguiente concepto:
El clima de servicio es la percepción de los empleados de las prácticas, procedimientos y comportamientos que se recompensan, es compatible y esperado con respecto al servicio al cliente y la calidad de servicio al cliente, por ejemplo, en la medida que los empleados perciban que son recompensados por la entrega de calidad de servicio, el clima de servicio de su organización será más fuerte (p. 151) [traducción nuestra].

El constructo planteado describe que "los trabajadores que perciben un clima de servicio alto presentan recursos necesarios para tratar con variabilidad al cliente (usuario interno) y tienen motivación para hacerlo, requieren menos supervisión y dan servicios de alta calidad" (Mayer, Ehrhart, y Schneider, 2009, p. 1036) [traducción nuestra] durante el proceso de la actividad.

De igual manera, los niveles de interdependencia deben ser elevados en una institución, para lo cual requiere de una cultura corporativa que propicie un clima de servicio alto, márgenes de libertad y un fortalecimiento de la relación. Por el contrario, un desglose de cualquiera de los empleados involucrados afecta negativamente la calidad del servicio, siendo importante que todos los trabajadores en la cadena de servicio comprendan la importancia de su labor en la fidelización y satisfacción del cliente (Reardon y Enis, 1990). Las evidencias empíricas señalan que existe relación entre las características de la organización y las actitudes de los empleados hacia la calidad del servicio, la satisfacción del cliente y su lealtad a los resultados financieros (Dean, 2004), esto resalta la importancia de la percepción del usuario interno como una premisa básica que refleja la calidad del servicio.

En tal sentido, para desarrollar la calidad de servicios se propicia la implementación de estrategias organizacionales que generen un clima donde el usuario interno al interactuar sienta que es parte de una institución. La motivación y la satisfacción del cliente interno ante las funciones que realiza diariamente lo orientan a tener una actitud positiva y entusiasta (Ospina, 2014). Así, se relaciona la satisfacción del trabajador en su centro de trabajo con la satisfacción del cliente que recibe el servicio, esto último acreditado por el proceso de un servicio exitoso en base a la calidad (Dean, 2004).

El proceso de gestión del clima de trabajo es importante pues modera la relación entre la percepción del empleado sobre el clima de servicio y la satisfacción del cliente (Schneider y Nicole, 2002), 
la relación positiva entre el clima de servicio y la percepción del cliente sobre la calidad del servicio y su satisfacción (Dean 2004), y la relación entre esa misma percepción de calidad y el rendimiento de la organización (Abedin, Rahman y Mohiuddin, 2016). A su vez, hay una relación significativa entre la satisfacción del cliente y el retorno de la inversión; sin embargo, este vínculo varía según la industria, el país, la categoría de negocio (Zeithaml, 2000) y el valor para los accionistas, entendido este último como una función del flujo de caja futuro y la reducción en la variabilidad del flujo efectivo en el tiempo (Gruca y Rego, 2005).

El desarrollo de una cultura de servicio corporativo está estrechamente relacionado con el marketing interno, el cual, actualmente, se considera un requisito para el éxito de la comercialización externa (Grönroos, 1985 y 1990). Su principal desafío estratégico es crear un entorno de trabajo marcado por tareas interesantes y -desde el punto de vista de los empleados - significativas, ya que se busca aumentar su satisfacción y reforzar su interés en la atención al cliente. Además, estudios demuestran que cada vez que un cliente interno se encuentra satisfecho con las políticas, normas y valores de la organización, así como con su puesto actual, su desarrollo profesional, entre otros aspectos, al hacer sus funciones prestará un servicio de calidad, no solo porque presentará mejor trato y amabilidad, sino también porque se mostrará más ágil y diligente, y será poseedor de una elevada autoestima, lo que le dará un mejor porte y aspecto (Guevara, 2009). Así, los factores contextuales que sostienen el comportamiento laboral constituyen un elemento necesario para el clima de servicio, mas no son suficientes.

Por ello, se deben implementar estrategias de gestión que desarrollen habilidades o competencias sociales que permitan a los clientes realizar su trabajo con parámetros de calidad de servicio (Schneider, White y Paul, 1998). También, para una buena estrategia de servicio al cliente, es necesaria la involucración de la institución, el liderazgo de la alta gerencia, la satisfacción, el conocimiento del valor del servicio, la productividad y la lealtad de los empleados (Ospina, 2014). El liderazgo de la gestión es de vital importancia en la generación de conductas motivadas del trabajador (Kolb, Rubin y Mclntyre, 1977), las cuales tienen, de alguna manera, influencia en el ofrecimiento de servicios de calidad.

Bajo la importancia de esos hallazgos, este artículo se centra en conocer la percepción del cliente interno (servidor civil) como parte de una investigación sobre el clima organizacional y la percepción de la calidad del servicio del cliente interno en el sector público. Proponemos que este sector debe fortalecer sus estrategias de gestión del talento humano en pro de brindar servicios de calidad que le permitan alcanzar los objetivos estratégicos institucionales. Por las razones expuestas, se atenderá a la percepción del cliente interno sobre la calidad del servicio y su valor como parte del logro de los objetivos institucionales de la entidad pública, a fin de crear conciencia sobre la importancia de fortalecer una cultura corporativa sólida en clima de servicio, incrementando así la motivación del trabajador y la satisfacción de la población al recibir servicios públicos. Considerando lo anterior, en este estudio nos proponemos despejar la siguiente pregunta: ¿cuáles son las características de los factores de la percepción de la calidad del servicio percibido por el usuario interno de una institución pública? En tal sentido, nuestro objetivo es determinar aquellas características.

\section{METODOLOGÍA}

La presente investigación utiliza un enfoque de tipo cuantitativo y de nivel descriptivo-comparativo, de diseño no experimental y transversal, y de método hipotético deductivo (Hernández, Fernández y Baptista, 2014).

La población analizada estuvo constituida por servidores de una institución del sector público del Poder Ejecutivo, como se aprecia en la Tabla 1:

Tabla 1. Distribución de la población.

\begin{tabular}{|c|c|c|c|c|}
\hline & CAS & Nombrado & Terceros & Total \\
\hline \multirow{2}{*}{ PEA } & 703 & 231 & 501 & 1435 \\
& $(48,99 \%)$ & $(16,09 \%)$ & $(34,92 \%)$ & $(100,00 \%)$ \\
\hline
\end{tabular}

Fuente: Institución de estudio.

La muestra estuvo conformada por 310 servidores, entre hombres y mujeres, con un margen de error del 0,05 y un nivel de confianza del 0,95. A la muestra se le aplicó el procedimiento de Cochrane, como se puede ver en la Tabla 2.

El instrumento utilizado fue la escala de percepción de la calidad de servicio del usuario interno (EPCSU-IP), con el análisis factorial confirmatorio 0,85 de Paredes y Hernández. Además, el presente estudio tiene una confiabilidad de 0,924.

\section{RESULTADOS}

\section{Análisis de fiabilidad}

Se realizó el análisis de fiabilidad de las dimensiones de percepción de la calidad del servicio del 
Tabla 2. Distribución de la muestra.

\begin{tabular}{|l|c|c|c|}
\hline \multirow{3}{*}{ Sexo } & & Cantidad (c) & Porcentaje (\%) \\
\hline \multirow{4}{*}{ Contratados con el Estado } & Hombre & 144 & 46,5 \\
\cline { 2 - 4 } & Mujer & 166 & 53,5 \\
\cline { 2 - 4 } & Nombrado & 58 & 18,7 \\
\cline { 2 - 4 } & CAS & 145 & 46,8 \\
\hline \multirow{3}{*}{ Rango de edad } & Tercero & 107 & 34,5 \\
\hline \multirow{3}{*}{ Años de trabajo en la institución } & Entre 20 y 39 años & 152 & 49,0 \\
\cline { 2 - 4 } & Entre 40 y 49 años & 81 & 26,1 \\
\cline { 2 - 4 } & Más de 50 años & 77 & 24,8 \\
\cline { 2 - 4 } & Entre 1 y 2 años & 67 & 21,6 \\
\cline { 2 - 4 } & Entre 3 y 5 años & 70 & 22,6 \\
\cline { 2 - 4 } & $>5$ años & 89 & 28,7 \\
\hline
\end{tabular}

$c=310$

Fuente: Elaboración propia.

Tabla 3. Análisis de fiabilidad.

\begin{tabular}{|c|c|}
\hline Factores & $\boldsymbol{\alpha}$ \\
\hline Cooperación entre usuarios internos & 0,836 \\
\hline Liderazgo de gestión & 0,856 \\
\hline Responsabilidad institucional & 0,741 \\
\hline Valoración de su labor & 0,779 \\
\hline Escala total & 0,924 \\
\hline
\end{tabular}

Fuente: Elaboración propia.

Tabla 4. Estadísticos descriptivos.

\begin{tabular}{|c|c|c|c|c|c|}
\hline & $\begin{array}{c}\text { Cooperación entre } \\
\text { usuarios internos }\end{array}$ & $\begin{array}{c}\text { Liderazgo de } \\
\text { la gestión }\end{array}$ & $\begin{array}{c}\text { Responsabilidad } \\
\text { social institucional }\end{array}$ & $\begin{array}{c}\text { Valoración de su } \\
\text { labor }\end{array}$ & $\begin{array}{c}\text { Total de percepción } \\
\text { del servicio }\end{array}$ \\
\hline Media & 3,67 & 3,45 & 3,27 & 3,73 & 3,54 \\
\hline Error típico & 0,04 & 0,05 & 0,04 & 0,03 & 0,03 \\
\hline Mediana & 3,8 & 3,75 & 3,4 & 3.8 & 3,63 \\
\hline Moda & 4 & 4 & 3,2 & 4 & 3,68 \\
\hline Desviación estándar & 0,66 & 0,88 & 0,66 & 0,60 & 0,59 \\
\hline Varianza de la muestra & 0,44 & 0,77 & 0,44 & 0,36 & 0,35 \\
\hline Coeficiente de asimetría & $-1,18$ & $-0,76$ & $-0,64$ & $-0,88$ & $-0,89$ \\
\hline Rango & 4 & 4 & 4 & 4 & 4 \\
\hline Mínimo & 1 & 1 & 1 & 1 & 1 \\
\hline Máximo & 5 & 5 & 5 & 5 & 5 \\
\hline Suma & 1139 & 1069 & 1014 & 3157 & 1096 \\
\hline Sujetos & 310 & 310 & 310 & & 310 \\
\hline
\end{tabular}

Fuente: Elaboración propia.

cliente interno mediante el alfa de Cronbach ( $\alpha$ ), como se aprecia en Tabla 3. En el cuadro, se observa que ninguna dimensión es menor a 0,7; por el contrario, hay dos dimensiones que son mayores a 0,8 , lo que quiere decir que, al hacer el análisis, las dimensiones tienen poco margen de error.
En la Tabla 4, se registra que la percepción de la calidad de servicio interno se ubica en el grado 3, percepción ambivalente $(3,54)$. Los factores que describen la escala están en grado 3 , con una tendencia a ser favorable en los factores de cooperación entre usuarios internos $(3,67)$ y en la valora- 
ción de su labor $(3,73)$. Asimismo, se muestra una tendencia negativa en los factores de liderazgo de la gestión $(3,45)$ y de responsabilidad social institucional $(3,27)$.

Para el análisis de normalidad se utilizó el estadístico de Kolmogorov-Smirnov, obteniéndose un resultado menor a 0,00 para todas las dimensiones de percepción de la calidad de servicio del usuario interno. Por ello, los puntajes no se distribuyeron normalmente y se tuvo que aplicar pruebas no paramétricas en el análisis comparativo.

\section{Análisis comparativo}

Para hacer la comparación según el sexo se utilizó el estadístico no paramétrico de $U$ de Mann-Whitney. En el resultado no se encontraron diferencias significativas $(p>0,05)$, por lo que podríamos decir que la percepción sobre la calidad de servicio del cliente interno entre hombres y mujeres no presenta distinciones significativas (ver Tabla 5).

Por otro lado, para comparar los tipos de contratación (nombrados, CAS y terceros) se utilizó el estadístico de Kruskal-Wallis $(p<0,05)$, encontrándose diferencias significativas en la cooperación entre usuarios internos ( $p=0,017)$, el liderazgo de la gestión $(p=0,00)$ y la valoración de su labor $(p=0,005)$. Estas distinciones, como se aprecia en la Tabla 6, exponen que el grupo de los terceros tiene mayor puntaje en cooperación, liderazgo y valoración frente a los nombrados y CAS. Con respecto a la responsabilidad social institucional, el régimen laboral no plantea diferencias significativas, los trabajadores de los tres regímenes laborales analizados se ubican en la categoría de ambivalencia.
Para comparar el rango de edad de los servidores se utilizó el estadístico de Kruskal-Wallis $(p<0,05)$, con lo cual se halló diferencias significativas en liderazgo $(p=0,018)$, especialmente entre los trabajadores más jóvenes (de 20 a 39 años), como se observa en la Tabla 7.

Para comparar los años de servicio en la institución se utilizó el estadístico de Kruskal-Wallis $(p<0,05)$, encontrándose diferencias significativas en el liderazgo $(p=0,00)$ y en la valoración de su labor $(p=0,045)$. Se percibió, además, mayor liderazgo para la gestión y valoración de su labor en los servidores que tienen menos de un año en la institución. Los factores de cooperación entre usuarios internos y responsabilidad social institucional no presentan diferencias significativas según los años de servicio en la institución, como se aprecia en la Tabla 8.

\section{DISCUSIÓN}

Esta investigación tiene por objetivo describir la percepción de la calidad de servicio del usuario interno en el sector público. El resultado obtenido evidencia que esta percepción es ambivalente $(3,54)$, tiene una tendencia a ser favorable en las dimensiones de cooperación entre usuarios internos $(3,67)$ y la valoración de su labor $(3,73)$; mientras que en los factores de liderazgo de la gestión $(3,45)$ y de responsabilidad social institucional $(3,27)$ muestra una tendencia negativa. Estas dimensiones desfavorables necesitan ser enmendadas dando mayor significación a los constructos de responsabilidad social institucional, con el fin de aumentar el comportamiento del servicio prosocial por el rol y la cooperación entre trabajadores (Bellou y Andronikidis,

Tabla 5. Análisis comparativo según sexo.

\begin{tabular}{|c|c|c|c|c|c|c|c|c|c|}
\hline Factores & Sexo & Cantidad (c) & Rango B & I rangos & $\mathbf{M}$ & $\begin{array}{c}\text { Desviación } \\
\text { estándar }\end{array}$ & $\mathbf{U}$ & $\mathbf{Z}$ & $\begin{array}{c}\text { Sig. asintótica } \\
\text { (bilateral) }\end{array}$ \\
\hline \multirow{2}{*}{ CUI } & $\mathrm{F}$ & 144 & 147,42 & 21228,00 & 3,61 & 0,70 & 10788,00 & $-1,495$ & 0,135 \\
\cline { 2 - 11 } & $\mathrm{M}$ & 166 & 162,51 & 26977,00 & 3,73 & 0,62 & & & \\
\hline \multirow{2}{*}{ LG } & $\mathrm{F}$ & 144 & 161,47 & 23251,50 & 3,50 & 0,87 & 11092,50 & $-1,099$ & 0,272 \\
\cline { 2 - 11 } & $\mathrm{M}$ & 166 & 150,32 & 24953,50 & 3,41 & 0,89 & & & \\
\hline \multirow{2}{*}{ RSI } & $\mathrm{F}$ & 144 & 156,11 & 22480,50 & 3,30 & 0,64 & 11863,50 & $-0,113$ & 0,910 \\
\cline { 2 - 11 } & $\mathrm{M}$ & 166 & 154,97 & 25724,50 & 3,25 & 0,68 & & & \\
\hline \multirow{2}{*}{ VL } & $\mathrm{F}$ & 144 & 152,59 & 21973,00 & 3,72 & 0,57 & 11533,00 & $-0,537$ & 0,591 \\
\cline { 2 - 10 } & $\mathrm{M}$ & 166 & 158,02 & 26232,00 & 3,74 & 0,62 & & & \\
\hline \multirow{2}{*}{$\begin{array}{c}\text { Total percepción } \\
\text { servicio }\end{array}$} & $\mathrm{F}$ & 144 & 154,02 & 22179,00 & 3,53 & 0,59 & 11739,00 & $-0,271$ & 0,787 \\
\cline { 2 - 10 } & $\mathrm{M}$ & 166 & 156,78 & 26026,00 & 3,54 & 0,60 & & &
\end{tabular}

$c=310$

CUI=Cooperación entre usuarios internos. LG=Liderazgo de la gestión. RSI=Responsabilidad social institucional. VL=Valoración de su labor. Fuente: Elaboración propia. 
Tabla 6. Análisis comparativo según el tipo de contratación.

\begin{tabular}{|c|c|c|c|c|c|c|c|c|}
\hline Factores & $\begin{array}{c}\text { Tipo de } \\
\text { contratación }\end{array}$ & Cantidad (c) & $\begin{array}{c}\text { Rango } \\
\text { Promedio }\end{array}$ & Media & $\begin{array}{l}\text { Desviación } \\
\text { estándar }\end{array}$ & $\chi^{2}$ & $\begin{array}{c}\text { Grados de } \\
\text { libertad }\end{array}$ & $\begin{array}{c}\text { Sig. } \\
\text { asintótica }\end{array}$ \\
\hline \multirow{4}{*}{ CUI } & Nombrado & 58 & 126,88 & 3,37 & 0,89 & & & \\
\hline & CAS & 145 & 158,18 & 3,73 & 0,55 & & & \\
\hline & Terceros & 107 & 167,39 & 3,77 & 0,62 & & & \\
\hline & Total & 310 & & 3,67 & 0,66 & 8,099 & 2 & 0,017 \\
\hline \multirow{4}{*}{ LG } & Nombrado & 58 & 125,29 & 3,13 & 1,00 & & & \\
\hline & CAS & 145 & 142,53 & 3,36 & 0,83 & & & \\
\hline & Terceros & 107 & 189,45 & 3,75 & 0,79 & & & \\
\hline & Total & 310 & & 3,45 & 0,88 & 25,298 & 2 & 0,000 \\
\hline \multirow{4}{*}{ RSI } & Nombrado & 58 & 139,21 & 3,09 & 0,81 & & & \\
\hline & CAS & 145 & 152,54 & 3,27 & 0,62 & & & \\
\hline & Terceros & 107 & 168,34 & 3,37 & 0,63 & & & \\
\hline & Total & 310 & & 3,27 & 0,66 & 4,316 & 2 & 0,116 \\
\hline \multirow{4}{*}{ VL } & Nombrado & 58 & 123,46 & 3,47 & 0,72 & & & \\
\hline & CAS & 145 & 157,24 & 3,77 & 0,51 & & & \\
\hline & Terceros & 107 & 170,51 & 3,83 & 0,60 & & & \\
\hline & Total & 310 & & 3,73 & 0,60 & 10,671 & 2 & 0,005 \\
\hline \multirow{4}{*}{$\begin{array}{l}\text { Total de percepción } \\
\text { del servicio }\end{array}$} & Nombrado & 58 & 123,67 & 3,27 & 0,75 & & & \\
\hline & CAS & 145 & 150,57 & 3,54 & 0,50 & & & \\
\hline & Terceros & 107 & 179,43 & 3,68 & 0,56 & & & \\
\hline & Total & 310 & & 3,54 & 0,59 & 15,400 & 2 & 0,000 \\
\hline
\end{tabular}

CUI=Cooperación entre usuarios internos. LG=Liderazgo de la gestión. RSI=Responsabilidad social institucional. VL=Valoración de su labor. Fuente: Elaboración propia.

Tabla 7. Análisis comparativo según periodo de edad cronológica.

\begin{tabular}{|c|c|c|c|c|c|c|c|c|}
\hline Edad & Promedio de edad & Cantidad & $\begin{array}{c}\text { Rango } \\
\text { promedio }\end{array}$ & $\mathbf{M}$ & $\begin{array}{l}\text { Desviación } \\
\text { estándar }\end{array}$ & 2 & $\begin{array}{l}\text { Grados de } \\
\text { libertad }\end{array}$ & $\begin{array}{c}\text { Sig. } \\
\text { asintótica }\end{array}$ \\
\hline \multirow{4}{*}{ CUI } & De 29 a 39 años & 152 & 161,80 & 3,74 & 0,57 & & & \\
\hline & De 40 a 49 años & 81 & 153,88 & 3,69 & 0,62 & & & \\
\hline & De 50 a más años & 77 & 144,77 & 3,52 & 0,84 & & & \\
\hline & Total & 310 & & 3,67 & 0,66 & 1,923 & 2 & 0,382 \\
\hline \multirow{4}{*}{ LG } & De 29 a 39 años & 152 & 169,82 & 3,59 & 0,81 & & & \\
\hline & De 40 a 49 años & 81 & 145,43 & 3,38 & 0,85 & & & \\
\hline & De 50 a más años & 77 & 137,84 & 3,24 & 1,00 & & & \\
\hline & Total & 310 & & 3,45 & 0,88 & 7,991 & 2 & 0,018 \\
\hline \multirow{4}{*}{ RSI } & De 29 a 39 años & 152 & 157,15 & 3,30 & 0,63 & & & \\
\hline & De 40 a 49 años & 81 & 157,10 & 3,29 & 0,64 & & & \\
\hline & De 50 a más años & 77 & 150,56 & 3,20 & 0,76 & & & \\
\hline & Total & 310 & & 3,27 & 0,66 & 0,315 & 2 & 0,854 \\
\hline \multirow{4}{*}{ VL } & De 29 a 39 años & 152 & 158,34 & 3,77 & 0,55 & & & \\
\hline & De 40 a 49 años & 81 & 166,40 & 3,80 & 0,57 & & & \\
\hline & De 50 a más años & 77 & 138,43 & 3,59 & 0,69 & & & \\
\hline & Total & 310 & & 3,73 & 0,60 & 4,224 & 2 & 0,121 \\
\hline \multirow{4}{*}{$\begin{array}{l}\text { Total de } \\
\text { percepción del } \\
\text { servicio }\end{array}$} & De 29 a 39 años & 152 & 162,07 & 3,60 & 0,54 & & & \\
\hline & De 40 a 49 años & 81 & 157,70 & 3,55 & 0,55 & & & \\
\hline & De 50 a más años & 77 & 140,21 & 3,40 & 0,72 & & & \\
\hline & Total & 310 & & 3,54 & 0,59 & 3,110 & 2 & 0,211 \\
\hline
\end{tabular}

CUI=Cooperación entre usuarios internos. LG=Liderazgo de la gestión. RSI=Responsabilidad social institucional. VL=Valoración de su labor. Fuente: Elaboración propia. 
Tabla 8. Análisis comparativo según los años de servicio en la institución.

\begin{tabular}{|c|c|c|c|c|c|c|c|c|}
\hline Factores & Antigüedad & $\begin{array}{l}\text { Cantidad } \\
\text { (c) }\end{array}$ & $\begin{array}{c}\text { Rango } \\
\text { promedio }\end{array}$ & M & $\begin{array}{l}\text { Desviación } \\
\text { estándar }\end{array}$ & $\chi^{2}$ & $\begin{array}{c}\text { Grados de } \\
\text { libertad }\end{array}$ & $\begin{array}{c}\text { Sig. } \\
\text { asintótica }\end{array}$ \\
\hline \multirow{5}{*}{ CUI } & >1 año & 67 & 175,31 & 3,8388 & 0,47640 & & & \\
\hline & Entre 1 y 2 años & 70 & 159,58 & 3,6629 & 0,72195 & & & \\
\hline & Entre 2 y 5 años & 89 & 144,66 & 3,6337 & 0,62394 & & & \\
\hline & Más de 5 años & 84 & 147,79 & 3,5976 & 0,76015 & & & \\
\hline & Total & 310 & & 3,6748 & 0,66250 & 5,461 & 3 & 0,141 \\
\hline \multirow{5}{*}{ LG } & $>1$ año & 67 & 199,40 & 3,8619 & 0,61435 & & & \\
\hline & Entre 1 y 2 años & 70 & 164,75 & 3,4821 & 0,97554 & & & \\
\hline & Entre 2 y 5 años & 89 & 134,94 & 3,3258 & 0,78462 & & & \\
\hline & Más de 5 años & 84 & 134,55 & 3,2262 & 0,96522 & & & \\
\hline & Total & 310 & & 3,4500 & 0,87965 & 26,422 & 3 & 0,000 \\
\hline \multirow{5}{*}{ RSI } & $>1$ año & 67 & 173,01 & 3,3910 & 0,60646 & & & \\
\hline & Entre 1 y 2 años & 70 & 161,85 & 3,2800 & 0,73929 & & & \\
\hline & Entre 2 y 5 años & 89 & 150,00 & 3,2652 & 0,62288 & & & \\
\hline & Más de 5 años & 84 & 142,07 & 3,1786 & 0,68303 & & & \\
\hline & Total & 310 & & 3,2723 & 0,66474 & 5,189 & 3 & 0,158 \\
\hline \multirow{5}{*}{ VL } & $>1$ año & 67 & 179,31 & 3,8985 & 0,47146 & & & \\
\hline & Entre 1 y 2 años & 70 & 160,27 & 3,7343 & 0,64040 & & & \\
\hline & Entre 2 y 5 años & 89 & 147,94 & 3,7079 & 0,60307 & & & \\
\hline & Más de 5 años & 84 & 140,54 & 3,6286 & 0,62721 & & & \\
\hline & Total & 310 & & 3,7335 & 0,59781 & 8,054 & 3 & 0,045 \\
\hline \multirow{5}{*}{$\begin{array}{l}\text { Total de } \\
\text { percepción del } \\
\text { servicio }\end{array}$} & $>1$ año & 67 & 188,34 & 3,7416 & 0,44156 & & & \\
\hline & Entre 1 y 2 años & 70 & 166,79 & 3,5429 & 0,68392 & & & \\
\hline & Entre 2 y 5 años & 89 & 140,43 & 3,4914 & 0,54644 & & & \\
\hline & Más de 5 años & 84 & 135,88 & 3,4173 & 0,63109 & & & \\
\hline & Total & 310 & & 3,5370 & 0,59303 & 16,669 & 3 & 0,001 \\
\hline
\end{tabular}

CUI=Cooperación entre usuarios internos. LG=Liderazgo de la gestión. RSI=Responsabilidad social institucional. VL=Valoración de su labor.

Fuente: Elaboración propia.

2008). Además, resulta favorable y significativo que un jefe apoye las nuevas ideas e iniciativas en la gestión de liderazgo por ser una institución burocrática, pues se tiende a creer que esta es limitada para generar contextos de interdependencia que propicien la creatividad y la variabilidad del comportamiento (Mayer, Ehrhart y Schneider, 2009; Dean, 2004; Paredes, 1997).

Por otro lado, es necesario considerar las condiciones laborales que ofrecen las instituciones públicas, específicamente la estabilidad laboral que producen, la cual genera un vínculo con la entidad. No obstante, tal como se evidenció en esta investigación, el factor anterior no es determinante frente el comportamiento orientado al buen servicio entre usuarios internos. Además, el personal que no tiene una estabilidad laboral, ni vínculo con la institución ("terceros"), presenta mayor puntaje en la percepción de la cooperación entre usuarios internos $(p=0,017)$, en la percepción del liderazgo de la gestión $(p=0,00)$ y en la percepción de la valoración de su labor $(p=0,005)$; ello frente a los nombrados y CAS, quienes aparentemente tienen una condición de estabilidad laboral que no presenta impacto en los factores estudiados. Asimismo, los empleadores que tienen menos años trabajando en la institución presentan un mayor nivel de percepción del liderazgo de la gestión $(p=0,00)$ y percepción de la valoración de su labor $(p=0,045)$, mientras que los más jóvenes (de 20 a 39 años) muestran mayor grado de percepción del liderazgo de la gestión $(p=0,018)$ en contraste con los mayores de edad. Estos hallazgos nos hacen reflexionar en torno a la mejora del tema abordado: es necesario romper barreras y paradigmas frente a los nuevos contextos sociales y globales que están atravesando las nuevas generaciones, donde la estabilidad laboral 
y los años de trabajo en una institución no garantizan que el trabajador brinde un buen servicio al usuario interno, hecho que tiene un impacto en la satisfacción del cliente final ("la población”) a partir de la percepción.

Por esa razón, se requiere implementar estrategias que permitan desarrollar una cultura basada en el clima de servicio, en la que se respire calidad en todos los trabajadores (nuevos y antiguos), ya que la fuerza del clima modera la relación entre la percepción del empleado sobre el clima de servicio y la satisfacción del cliente (Schneider y Nicole, 2002). Asimismo, las organizaciones que propician una cultura basada en la calidad del servicio, mediante los servidores que cumplan sus funciones, ofrecerán no solo un servicio, sino también calidad del mismo, sobrepasando las expectativas de la población (Simancas, 2012).

Las evidencias encontradas ubican a la percepción de la calidad de servicio del usuario interno, con una direccionalidad no precisa, a ser positiva o negativa; ambivalencia que se le atribuye a los factores de tendencia negativa que se han observado en la gestión de liderazgo y responsabilidad social institucional, las mismas que deben ser estudiadas con el fin de lograr mejores estándares del desempeño en el proceso de gestión y en el bienestar personal.

\section{CONCLUSIONES}

1. La percepción de la calidad de servicio en servidores de una institución del sector público es ambivalente, con una tendencia a ser favorable en los factores de percepción de la cooperación entre usuarios internos y de la valoración de su labor.

2. En relación a los tipos de contratación (nombrados, CAS y terceros), se evidencian diferencias significativas en los factores de percepción de la cooperación entre usuarios internos, del liderazgo de la gestión y de la valoración de su labor, siendo el grupo de terceros los que tienen un mayor nivel de percepción positiva frente a los nombrados y CAS.

3. Los trabajadores más jóvenes (de 20 a 39 años) presentan un mayor grado de percepción en el factor liderazgo de la gestión frente a los mayores de edad.

4. En relación a los años de servicio en la institución, se aprecian diferencias significativas en la percepción de los factores de liderazgo de la gestión y de valoración de su labor, obser- vándose una mayor percepción positiva en los trabajadores que tienen menos de un año en la institución, en contraste a los que tienen mayor tiempo de servicio.

\section{REFERENCIAS BIBLIOGRÁFICAS}

[1] Abedin, Z., Rahman, M. y Mohiuddin, L. (2016). Service Quality Level and the Perception of Customers: Astudy on Nijhoom Tours $-5^{*}$ Rated Travel and Tourism Company in Bangladesh. International Journal of Management Sciences and Business Research, 5(11), 109-118.

[2] Adler, A. (1973). Superiority and Social Interest: A Collection of Later Writings. Nueva York, Estados Unidos: Viking Press.

[3] Argyris, C. (1973). Personality and Organization Theory Revisited. Administrative Science Quarterly, 18(2), 141-167.

[4] Balmori, G. y Flores, J. B. (2014). MECSI: Modelo para evaluar la calidad del servicio interno. Innovaciones de Negocios, 11(22), 191-213.

[5] Bellou, V. y Andronikidis, A. (2008). The impact of internal service quality on customer service behaviour: Evidence from the banking sector. International Journal of Quality \& Reliability Management, 25(9), 943-954.

[6] Dean, A. M. (2004). Links between organisational and customer variables in services delivery: Evidence, contradictions and challenges. International Journal of Service Industry Management, 15(4), 332-350.

[7] Domínguez, H. (2006). El servicio invisible. Fundamento de un buen servicio al cliente. Colombia: ECOE Ediciones.

[8] Grönroos, C. (1984). A Service Quality Model and its Marketing Implications. European Journal of Marketing, 18(4), 36-44.

[9] Grönroos, C. (1985). Internal Marketing Theory and Practice. En T. M. Bloch, H. \& R. Block, G. D. Upah, et al. (eds.), Services Marketing in a Changing Environment (pp. 41-47). Chicago. Estados Unidos: American Marketing Association.

[10] Grönroos, C. (1990). Relationship Approach to Marketing in Service Contexts: The Marketing and Organizational Behavior Interface. Journal of Bussines Research, (20), 3-11

[11] Guevara, U. L. de (2009). Medición de la satisfacción del cliente interno en una empresa 
de transformación. (Tesis de maestria). Universidad Veracruzana, México.

[12] Gruca, T. S. y Rego, L. (2005). Customer Satisfaction, Cash Flow, and Shareholder Value. Journal of Marketing, 69(3), 115-130.

[13] Hernández, R., Fernández, C. y Baptista, P. (2014). Metodología de la investigación (6 ed.). México D. F., México: Mc Graw Hill Education.

[14] Kolb, D. A., Rubin, I. M. y Mclntyre, J. M. (1977). Psicología de las organizaciones. Experiencias. Madrid, España: Ediciones del Castillo.

[15] Lapassade, G. (1977). Grupos, organizaciones e instituciones. La transformación de la burocracia. Barcelona, España: Gedisa.

[16] Mayer, D. M., Ehrhart, M. G. y Schneider, B. (2009). Service Attribute Boundary Conditions of the Service Climate-Customer Satisfaction Link. Academy of Management Journal, 52(5), 1034-1050.

[17] Miguel, J. M. de (1999). La organización como construcción social. Representación organizacional. (Tesis de doctorado). Universidad Autónoma de Madrid, España.

[18] Ospina, A. V. (2014). ¿Qué tan importante es el servicio al cliente interno y externo en una compañia? Recuperado de: https://repository. unimilitar.edu.co/bitstream/10654/13138/1/ ASTRID $\% 20$ VANESSA $\% 200$ SPINA $\% 20$ MAHECHA.pdf.

[19] Paredes, M. T. (1997). La interdependencia empresarial: factor de calidad en la gestión Caso sector empresarial pesquero peruano. (Tesis de maestría). Universidad Nacional Mayor de San Marcos, Lima.

[20] Reardon, K. K. y Enis, B. (1990). Establishing a Companywide Customer Orientation through Persuasive Internal Marketing. Management Communication Quarterly, 3(3), 376-387.

[21] Schneider, B. y Nicole, A. N. (2002). Climate Strength: ANew Direction for Climate Research. Journal of Applied Psychology, 87(2), 220-229.

[22] Schneider, B., White, S. S. y Paul, M. C. (1998). Linking Service Climate and Customer Perceptions of Service Quality: Test of a Causal Model. Journal of Applied Psychology, 83(2), 150-163.

[23] Simancas, R. (2012). Cliente interno y calidad de servicio en las organizaciones productivas. Dictamen Libre, (10-11), 81- 89.

[24] Zeithaml, V. A. (2000). Service Quality, Profitability, and the Economic Worth of Customer: What We Know and What We Need to Learn. Journal of the Academy of Marketing Science, 28(1), 67-85. 


\section{Internal user perception of service quality in a public institution}

Suseyla Roxana Sarmiento Prieto ${ }^{1}$ Mildred Teresa Paredes Tarazona ${ }^{2}$

\begin{abstract}
This is a quantitative, descriptive and comparative study conducted on a sample of 310 public servants that attempts to describe the perception of service quality of the internal user via the following factors: cooperation between internal users, leadership of management, corporate social responsibility and performance selfappraisal. This study defines the internal user as any collaborator holding a position in the organizational structure. The valid and reliable five-degree EPCSUIP ordinal scale was used for this research. The total perception is at a neutral level (3.54) with a tendency to higher degrees; it tends to be favorable in the dimensions of cooperation between internal users (3.67) and performance self-appraisal (3.73), and unfavorable regarding the factors leadership of management (3.45) and corporate social responsibility (3.27). The variable is studied as an indicator of the organizational climate in relation to the management of production processes of goods and services.
\end{abstract}

Keywords: Service quality; internal user; management; corporate social responsibility; organizational climate.

\section{INTRODUCTION}

Public institutions in Peru, as bureaucratic organizations, devote and focus their interest on regulatory compliance and oversight of activities performed (Lapassade, 1977). Over the years, due to the nature of the social construction of these organizations (Miguel, 1999), the quality of service provided to external users has been neglected, causing discomfort among the population that describes it as slow, poor, bureaucratic, corrupt and incompetent. This discontent is expressed in the increase of complaints from external customers. At the same time, internal customers lack interest in providing good service.

Most of the output and efforts are focused on the external user or customer, in order to achieve customer loyalty, among other reasons; however, the internal user or internal customer is neglected (Balmori \& Flores, 2014). This situation tends to affect institutional processes when not addressed by management, because internal users are not aware that they provide a service to their co-workers as well, favorably and efficiently impacting their performance. As a result, workers forget their role as internal providers of their colleagues and other bodies within the institution.

Internal users are seen as strategic allies within non-profit organizations and institutions, due to the fact that they are the bedrock of the group. They increase revenues and position the business through their products and services (Domínguez, 2006). They are also the artifice in the achievement of strategic objectives, given that they will "ofrecerán no solo un servicio sino una calidad de servicio que logre sobrepasar las expectativas del cliente externo, llamar su atención, lograr esa fidelización que la empresa necesita y despertar el deseo del cliente de volver" [offer not only a service but a quality service that exceeds the external customers' expectations, draws their attention, gains customer loyalty that the company needs, and sparks their desire to return] (Simancas, 2012, p. 89).

It is therefore important to understand that internal users interact in different ways when performing their functions, which is

1 Psychologist, General manager of Sugrafica at Inversiones Sujuro S. A. C., Lima, Peru. E-mail: ssarmiento@sugrafica.com

$2 \mathrm{PhD}$. in Psychology, professor at the School of Psychology of the Universidad Nacional Mayor de San Marcos. Lima, Peru.

E-mail: mparedest@unmsm.edu.pe 
evidenced by individual and group behaviors that, in general, are not aimed at providing the external customer with good service. This is evidenced in contrast to organizations that promote a working environment based on positive service that strengthens workers' behavior towards good customer service (Schneider, White \& Paul, 1998). Said variability in the employees' behavior is related to climate strength, and the latter is related to customer experience (Schneider \& Nicole, 2002), a fact that is reflected in a mature personality (Argyris, 1973). Internal service quality has a positive impact on prosocial behaviors via the role and cooperation among co-workers, leading to the conclusion that the provision of high quality internal services maintains or increases customer satisfaction (Bellou \& Andronikis, 2008). These prosocial behaviors are the reason for managing corporate social responsibility, given that by playing a significant role in the internal user's personal development through service valued on the basis of customer satisfaction, more than a simple commercial marketing practice is achieved.

The internal customer, agent of change and promotor and actor of production, requires certain conditions to enthusiastically perform his role to his full potential, aligning his expectations with the strategic objectives of the institution. This translates into cooperation and participation behaviors among internal users, organizational behaviors that enhance the ability to resolve issues and offer collective services based on a feeling of belonging to a community (Adler, 1973).

Service quality perception is "the outcome of an evaluation process, where the consumer compares his expectations with the service he perceives he has received...the result of this process will be the perceived quality of the service" (Grönoos, 1984, p. 37). For this reason, the customer makes a value judgment based on the expectations he originally had (Balmori \& Flores, 2014). Internal customer satisfaction is founded on an entire process, considering that the consumer (internal user) is not only interested in the product or service, but in the whole process (Grönroos, 1984), which involves other factors that create a pleasant experience during the delivery of the service. The following concept is suggested by Schneider, White and Paul (1998):

Climate for service refers to employee perceptions of the practices, procedures, and behaviors that get rewarded, supported, and expected with regard to customer service and customer service quality. For example, to the extent that employees perceive that they are rewarded for delivering quality service, their organization's service climate will be stronger (p. 151).

The posed construct outlines that "employees in units with a "high service climate" are surrounded by the resources required to deal with customer variability and have the motivation to do so, with the result that they require less direct supervision yet produce effective delivery of superior service quality" (Mayer, Ehrhart, \& Schneider, 2009, p. 1036) during the execution of the activity.

Similarly, levels of interdependence within an institution must be high, for which an institutional culture that fosters a high quality service climate, margins of freedom, and the strengthening of the relationship is required. A breakdown of any involved employee, however, has a negative impact on service quality, thus it is crucial that all workers in the service chain understand the importance of their role in building customer loyalty and satisfaction (Reardon \& Enis, 1990). Empirical evidence indicates that there is a link between organizational characteristics and employee attitudes expressed in service quality, customer satisfaction and loyalty, and profit (Dean, 2004). This highlights the importance of internal customers' perception as a basic premise that reflects service quality.

To that effect, implementation of organizational strategies that create a climate where the internal user, as he interacts with co-workers, feels like he is part of the institution is promoted. Internal user motivation and satisfaction about his day-to-day functions lead the employee to a positive and enthusiastic attitude (Ospina, 2014). Thus, employee satisfaction in the workplace is linked to the satisfaction of the costumer who receives the service, a fact that is confirmed by the process of a successful service based on quality (Dean, 2004).

The process management of work climate is important, because it moderates the relationship between employee perceptions and customer satisfaction (Schneider \& Nicole, 2002), the positive link between service climate and customer perception of service quality and customer satisfaction (Dean, 2004), and the relationship between that same quality perception and financial performance of the organization (Abedin, Rahman \& Mohiuddin, 2016). At the same time, there is a significant relationship between customer satisfaction and return on investment; however, this link varies depending on industry, country, business category (Zeithaml, 2000) and shareholder value, understanding the latter as a 
function of future cash flow and cash flow reduction over time (Gruca \& Rego, 2005).

The development of an institutional service culture is closely related to internal marketing, which is currently considered a requirement for successful external marketing (Grönroos, 1987 \& 1990). The main strategic challenge is creating a working environment characterized by interesting and significant tasks from the employees' perspective, as the aim is to increase their satisfaction and reinforce their interest in customer service. In addition, studies show that whenever an internal customer is satisfied with the organization's policies, norms and values, his current position, and his career development, among others, he will provide quality service. The employee not only will provide a better treatment and display kindness, but he will be agile, diligent, and will have high self-esteem, which will give him a better demeanor (Guevara, 2009). Thus, contextual factors that support work behavior are necessary elements for service climate, however, they are not enough.

Therefore, management strategies that provide customers with social skills and competences that allow them to perform their work considering service quality parameters should be implemented (Schneider, White \& Paul, 1998). Institution involvement, senior management leadership, satisfaction, understanding of service value, productivity and employee loyalty are necessary to create a good customer service strategy (Ospina, 2014). Management leadership is of crucial importance in the generation of motivated behaviors in the employee (Kolb, Rubin \& Mclntyre, 1977), which, to some extent, have an influence on the provision of quality services.

In view of the importance of these findings, this article is focused on learning more about internal customer (public servant) perception as part of a study on organizational climate and service quality perception of the internal customer in the public sector. We proposed that said sector reinforce its human talent management strategies in the interest of providing quality services that enable the achievement of organizational strategic objectives. For the reasons set forth above, internal customer perception regarding service quality and its value as part of the realization of institutional objectives of the public entity will be analyzed, in order to raise awareness about the importance of strengthening a robust institutional culture with a strong service climate, thereby increasing employee motivation and public satisfaction upon receiving public services. Based on the above, this study intends to answer the following question: what are the characteristics of service quality perception factors perceived by the internal customer of a public institution? In this sense, our objective is to determine those characteristics.

\section{METHODOLOGY}

Aquantitative, descriptive and comparative approach, a non-experimental and cross-sectional design, and the hypothetico-deductive method were used in this study (Hernández, Fernández \& Baptista, 2014).

The studied population consisted of staff members of a public sector institution of the Peruvian Executive Branch, as shown in Table 1:

Table 1. Population distribution.

\begin{tabular}{|c|c|c|c|c|}
\hline & CAS $^{\text {TN1 }}$ & Permanent & Outsourced & Total \\
\hline \multirow{2}{*}{ EAP } & 703 & 231 & 501 & 1435 \\
& $(48.99 \%)$ & $(16.09 \%)$ & $(34.92 \%)$ & $(100.00 \%)$ \\
\hline
\end{tabular}

Source: Institution studied.

The sample consisted of 310 public servants, male and female, with an error margin of 0.05 and a confidence level of 0.95 . The Cochrane-Orcutt estimation was applied to the sample, as shown in Table 2:

The internal user perception of service quality scale (EPCSU-IP) was the instrument used, with Paredes and Hernández factor analysis of 0.85 . This study has a reliability coefficient of 0.924 .

\section{RESULTS}

\section{Reliability Analysis}

The reliability analysis for the internal customer perception of service quality dimensions was performed using Cronbach's alpha $(\alpha)$, as shown in Table 3. It can be observed that no dimension coefficient is less than 0.7 , and on the contrary, two dimension coefficients are greater than 0.8 , implying there is little margin of error.

Table 4 indicates that internal service quality perception is at level 3 , indicating ambivalent perception (3.54). Factors describing the scale are placed at level 3, and a favorable trend was observed in cooperation among internal users (3.67) and performance self-appraisal (3.54) factors. A negative trend is observed in management leadership (3.45) and corporate social responsibility (3.27) factors.

TN1: Contract modality exclusive of the government that links a public entity with a natural person who provides a non-autonomous, subordinate and dependent service. 
Table 2. Sample distribution.

\begin{tabular}{|l|l|l|l|}
\hline \multirow{3}{*}{ Sex } & & Number (n) & Percentage (\%) \\
\hline \multirow{4}{*}{ Employed by the government } & Male & 144 & 46.5 \\
\cline { 2 - 4 } & Female & 166 & 53.5 \\
\hline \multirow{4}{*}{ Age range } & Permanent & 58 & 18.7 \\
\cline { 2 - 4 } & CAS & 145 & 46.8 \\
\cline { 2 - 4 } & Outsourced & 107 & 34.5 \\
\hline \multirow{5}{*}{ Years of work at the institution } & 20 to $39 \mathrm{y} / \mathrm{o}$ & 152 & 49.0 \\
\cline { 2 - 4 } & 19 to $49 \mathrm{y} / \mathrm{o}$ & 81 & 26.1 \\
\cline { 2 - 4 } & Over $50 \mathrm{y} / \mathrm{o}$ & 77 & 24.8 \\
\cline { 2 - 4 } & $<1 \mathrm{yr}$ & 67 & 21.6 \\
\cline { 2 - 4 } & 1 to $2 \mathrm{yr}$ & 70 & 22.6 \\
\cline { 2 - 4 } & 3 to $5 \mathrm{yr}$ & 89 & 28.7 \\
\cline { 2 - 4 } & $>5 \mathrm{yr}$ & 84 & 27.1 \\
\hline
\end{tabular}

$\mathrm{n}=310$

Source: Prepared by the authors.

Table 3. Reliability analysis.

\begin{tabular}{|c|c|}
\hline Factors & $\alpha$ \\
\hline Cooperation among internal users & 0.836 \\
\hline Management leadership & 0.856 \\
\hline Corporate social responsibility & 0.741 \\
\hline Performance self-appraisal & 0.779 \\
\hline Total scale & 0.924 \\
\hline
\end{tabular}

Source: Prepared by the authors.

Table 4. Descriptive statistics.

\begin{tabular}{|c|c|c|c|c|c|}
\hline & $\begin{array}{c}\text { Cooperation among } \\
\text { internal users }\end{array}$ & $\begin{array}{c}\text { Management } \\
\text { leadership }\end{array}$ & $\begin{array}{c}\text { Corporate social } \\
\text { responsibility }\end{array}$ & $\begin{array}{c}\text { Performance } \\
\text { self-appraisal }\end{array}$ & $\begin{array}{c}\text { Total perception } \\
\text { of service quality }\end{array}$ \\
\hline Mean & 3.67 & 3.45 & 3.27 & 3.73 & 3.54 \\
\hline Typical error & 0.04 & 0.05 & 0.04 & 0.03 & 0.03 \\
\hline Median & 3.8 & 3.75 & 3.4 & 3.8 & 3.63 \\
\hline Mode & 4 & 4 & 3.2 & 4 & 3.68 \\
\hline Standard deviation & 0.66 & 0.88 & 0.66 & 0.60 & 0.59 \\
\hline Sample variance & 0.44 & 0.77 & 0.44 & 0.36 & 0.35 \\
\hline Asymmetry coefficient & -1.18 & -0.76 & -0.64 & -0.88 & -0.89 \\
\hline Range & 4 & 4 & 4 & 4 & 4 \\
\hline Minimum & 1 & 1 & 1 & 1 & 1 \\
\hline Maximum & 5 & 5 & 5 & 5 & 5 \\
\hline Sum & 1139 & 1069 & 1014 & 1157 & 1096 \\
\hline Sample & 310 & 310 & 310 & 310 & 310 \\
\hline
\end{tabular}

Source: Prepared by the authors.

The Kolmogorov-Smirnov statistic was used for the normality analysis, obtaining a result of less than 0.00 for all the dimensions of the internal user perception of service quality. Therefore, scores were not normally distributed and nonparametric testing was required for the comparative analysis.

\section{Comparative analysis}

The Nonparametric Mann-Whitney $U$ test was used for the comparative analysis according to sex. No significant differences were found $(p>0.05)$, therefore it can be said that there is no significant distinc- 
tion in the internal costumer perception of quality service of men and women (see Table 5).

On the other hand, the Kruskal-Wallis statistic $(p<0.05)$ was used to compare contract modalities (permanent, CAS and outsourced). Significant differences were found in cooperation among internal users $(p=0.017)$, management leadership $(p=0.00)$, and performance self-appraisal $(p=0.005)$. As shown in Table 6, these differences indicate that the outsourced employees group has the highest scores in cooperation, leadership and performance self-appraisal compared to the permanent and CAS groups. Contract modalities do not pose significant differences regarding corporate social responsibility, workers of the three analyzed modalities are in the category of ambivalence.

The Kruskal-Wallis statistic $(p<0.05)$ was used to compare the age range of public servants, which revealed significant differences in leadership $(p=0.018)$, particularly among the youngest employees (20 to 39 years old), as shown in Table 7 .

The Kruskal-Wallis statistic $(p<0.05)$ was used to compare the number of years of work at the institution. Significant differences were found in leadership $(p=0.00)$ and performance self-appraisal $(p=0.045)$. In addition, greater management leadership and performance self-appraisal was observed in public servants that have less than a year working at the institution. No significant differences were found in cooperation among internal users and corporate social responsibility factors according to the years of work at the institution, as shown in Table 8:

\section{DISCUSSION}

This study aims to describe the internal user perception of quality service in the public sector. The obtained result demonstrated this perception is ambivalent (3.54), with a favorable trend in cooperation among internal users (3.67) and performance self-appraisal (3.73) dimensions; meanwhile a negative trend was observed in management leadership (3.45) and corporate social responsibility (3.27) factors. These unfavorable dimensions need to be amended by conferring greater significance to institutional social responsibility constructs, in order to enhance prosocial behaviors via the role and cooperation among co-workers (Bellou \& Andronikidis, 2008). In addition, it is positive and meaningful in management leadership that a management employee supports new ideas and initiatives in a bureaucratic institution, as it generally thought that the generation of interdependence contexts that favor creativity and behavior variability is limited in this type of institution (Mayer, Ehrhart \& Schneider, 2009; Dean, 2004; Paredes, 1997).

At the same time, it is necessary to consider the working conditions offered by public institutions, specifically, job security that creates a link between employees and institutions. However, as was evidenced in this research, the previous factor does not determine the workers' behavior towards a good service among internal customers. In addition, the personnel that have no job security nor links to the institution ("outsourced") obtained the highest scores regarding perception of cooperation among internal users $(p=0.017)$, management leadership $(p=0.00)$ and performance self-apprais-

Table 5. Comparative analysis according to sex.

\begin{tabular}{|c|c|c|c|c|c|c|c|c|c|}
\hline Factors & Sex & Number (n) & Range B & I ranges & $\mathbf{M}$ & $\begin{array}{c}\text { Standard } \\
\text { deviation }\end{array}$ & $\mathbf{U}$ & $\mathbf{Z}$ & $\begin{array}{c}\text { Asymptotic signifi- } \\
\text { cance (2-sided) }\end{array}$ \\
\hline \multirow{2}{*}{ CIU } & $\mathrm{F}$ & 144 & 147.42 & 21228.00 & 3.61 & 0.70 & 10788.00 & -1.495 & 0.135 \\
\cline { 2 - 11 } & $\mathrm{M}$ & 166 & 162.51 & 26977.00 & 3.73 & 0.62 & & & \\
\hline \multirow{2}{*}{ ML } & $\mathrm{F}$ & 144 & 161.47 & 23251.50 & 3.50 & 0.87 & 11092.50 & -1.099 & 0.272 \\
\cline { 2 - 11 } & $\mathrm{M}$ & 166 & 150.32 & 24953.50 & 3.41 & 0.89 & & & \\
\hline \multirow{2}{*}{ CSR } & $\mathrm{F}$ & 144 & 156.11 & 22480.50 & 3.30 & 0.64 & 11863.50 & -0.113 & 0.910 \\
\cline { 2 - 11 } & $\mathrm{M}$ & 166 & 154.97 & 25724.50 & 3.25 & 0.68 & & & \\
\hline \multirow{2}{*}{ PSA } & $\mathrm{F}$ & 144 & 152.59 & 21973.00 & 3.72 & 0.57 & 11533.00 & -0.537 & 0.591 \\
\cline { 2 - 10 } & $\mathrm{M}$ & 166 & 158.02 & 26232.00 & 3.74 & 0.62 & & & \\
\hline $\begin{array}{c}\text { Total service } \\
\text { quality }\end{array}$ & $\mathrm{F}$ & 144 & 154.02 & 22179.00 & 3.53 & 0.59 & 11739.00 & -0.271 & \\
\cline { 2 - 10 } & $\mathrm{M}$ & 166 & 156.78 & 26026.00 & 3.54 & 0.60 & & & \\
\hline
\end{tabular}

$\mathrm{n}=310$

$\mathrm{CIU}=$ Cooperation among internal users. ML=Management leadership. CSR=Corporate social responsibility. $P S A=P$ erformance self-appraisal. Source: Prepared by the authors. 
Table 6. Comparative analysis according to contract modality.

\begin{tabular}{|c|c|c|c|c|c|c|c|c|}
\hline Factors & $\begin{array}{l}\text { Contract } \\
\text { modality }\end{array}$ & Number (n) & $\begin{array}{c}\text { Average } \\
\text { range }\end{array}$ & Mean & $\begin{array}{l}\text { Standard } \\
\text { deviation }\end{array}$ & $\chi^{2}$ & $\begin{array}{l}\text { Degrees of } \\
\text { freedom }\end{array}$ & $\begin{array}{l}\text { Asymptotic } \\
\text { significance }\end{array}$ \\
\hline \multirow{4}{*}{ CIU } & Permanent & 58 & 126.88 & 3.37 & 0.89 & & & \\
\hline & CAS & 145 & 158.18 & 3.73 & 0.55 & & & \\
\hline & Outsourced & 107 & 167.39 & 3.77 & 0.62 & & & \\
\hline & Total & 310 & & 3.67 & 0.66 & 8.099 & 2 & 0.017 \\
\hline \multirow{4}{*}{ ML } & Permanent & 58 & 125.29 & 3.13 & 1.00 & & & \\
\hline & CAS & 145 & 142.53 & 3.36 & 0.83 & & & \\
\hline & Outsourced & 107 & 189.45 & 3.75 & 0.79 & & & \\
\hline & Total & 310 & & 3.45 & 0.88 & 25.298 & 2 & 0.000 \\
\hline \multirow{4}{*}{ CSR } & Permanent & 58 & 139.21 & 3.09 & 0.81 & & & \\
\hline & CAS & 145 & 152.54 & 3.27 & 0.62 & & & \\
\hline & Outsourced & 107 & 168.34 & 3.37 & 0.63 & & & \\
\hline & Total & 310 & & 3.27 & 0.66 & 4.316 & 2 & 0.116 \\
\hline \multirow{4}{*}{ PSA } & Permanent & 58 & 123.46 & 3.47 & 0.72 & & & \\
\hline & CAS & 145 & 157.24 & 3.77 & 0.51 & & & \\
\hline & Outsourced & 107 & 170.51 & 3.83 & 0.60 & & & \\
\hline & Total & 310 & & 3.73 & 0.60 & 10.671 & 2 & 0.005 \\
\hline \multirow{4}{*}{$\begin{array}{l}\text { Total service quality } \\
\text { perception }\end{array}$} & Permanent & 58 & 123.67 & 3.27 & 0.75 & & & \\
\hline & CAS & 145 & 150.57 & 3.54 & 0.50 & & & \\
\hline & Outsourced & 107 & 179.43 & 3.68 & 0.56 & & & \\
\hline & Total & 310 & & 3.54 & 0.59 & 15.400 & 2 & 0.000 \\
\hline
\end{tabular}

$\mathrm{CIU}=$ Cooperation among internal users. ML=Management leadership. CSR=Corporate social responsibility. PSA=Performance self-appraisal. Source: Prepared by the authors.

Table 7. Comparative analysis according to chronological age.

\begin{tabular}{|c|c|c|c|c|c|c|c|c|}
\hline Factors & Age range & Number & $\begin{array}{c}\text { Average } \\
\text { range }\end{array}$ & M & $\begin{array}{l}\text { Standard } \\
\text { deviation }\end{array}$ & $\chi^{2}$ & $\begin{array}{l}\text { Degrees of } \\
\text { freedom }\end{array}$ & $\begin{array}{l}\text { Asymptotic } \\
\text { significance }\end{array}$ \\
\hline \multirow{4}{*}{ CIU } & 29 to $39 \mathrm{y} / \mathrm{o}$ & 152 & 161.80 & 3.74 & 0.57 & & & \\
\hline & 40 to $49 \mathrm{y} / \mathrm{o}$ & 81 & 153.88 & 3.69 & 0.62 & & & \\
\hline & over 50 y/o & 77 & 144.77 & 3.52 & 0.84 & & & \\
\hline & Total & 310 & & 3.67 & 0.66 & 1.923 & 2 & 0.382 \\
\hline \multirow{4}{*}{ ML } & 29 to $39 \mathrm{y} / \mathrm{o}$ & 152 & 169.82 & 3.59 & 0.81 & & & \\
\hline & 40 to $49 \mathrm{y} / \mathrm{o}$ & 81 & 145.43 & 3.38 & 0.85 & & & \\
\hline & over $50 \mathrm{y} / \mathrm{o}$ & 77 & 137.84 & 3.24 & 1.00 & & & \\
\hline & Total & 310 & & 3.45 & 0.88 & 7.991 & 2 & 0.018 \\
\hline \multirow{4}{*}{ CSR } & 29 to $39 \mathrm{y} / \mathrm{o}$ & 152 & 157.15 & 3.30 & 0.63 & & & \\
\hline & 40 to $49 \mathrm{y} / \mathrm{o}$ & 81 & 157.10 & 3.29 & 0.64 & & & \\
\hline & over 50 y/o & 77 & 150.56 & 3.20 & 0.76 & & & \\
\hline & Total & 310 & & 3.27 & 0.66 & 0.315 & 2 & 0.854 \\
\hline \multirow{4}{*}{ PSA } & 29 to $39 \mathrm{y} / \mathrm{o}$ & 152 & 158.34 & 3.77 & 0.55 & & & \\
\hline & 40 to $49 \mathrm{y} / \mathrm{o}$ & 81 & 166.40 & 3.80 & 0.57 & & & \\
\hline & over 50 y/o & 77 & 138.43 & 3.59 & 0.69 & & & \\
\hline & Total & 310 & & 3.73 & 0.60 & 4.224 & 2 & 0.121 \\
\hline \multirow{4}{*}{$\begin{array}{l}\text { Total service } \\
\text { quality } \\
\text { perception }\end{array}$} & 29 to $39 \mathrm{y} / \mathrm{o}$ & 152 & 162.07 & 3.60 & 0.54 & & & \\
\hline & 40 to $49 \mathrm{y} / \mathrm{o}$ & 81 & 157.70 & 3.55 & 0.55 & & & \\
\hline & over 50 y/o & 77 & 140.21 & 3.40 & 0.72 & & & \\
\hline & Total & 310 & & 3.54 & 0.59 & 3.110 & 2 & 0.211 \\
\hline
\end{tabular}

$\mathrm{CIU}=$ Cooperation among internal users. $\mathrm{ML}=$ Management leadership. $\mathrm{CSR}=$ Corporate social responsibility. PSA=Performance self-appraisal. Source: Prepared by the authors. 
Table 8. Comparative analysis according to the number of years working at the institution.

\begin{tabular}{|c|c|c|c|c|c|c|c|c|}
\hline Factors & Seniority & Number (n) & Average range & $\mathbf{M}$ & $\begin{array}{l}\text { Standard } \\
\text { deviation }\end{array}$ & $\chi^{2}$ & $\begin{array}{c}\text { Degrees of } \\
\text { freedom }\end{array}$ & $\begin{array}{l}\text { Asymptotic } \\
\text { significance }\end{array}$ \\
\hline \multirow{5}{*}{ CIU } & $>1$ year & 67 & 175.31 & 3.8388 & 0.47640 & & & \\
\hline & 1 to $2 \mathrm{yr}$ & 70 & 159.58 & 3.6629 & 0.72195 & & & \\
\hline & 2 to $5 \mathrm{yr}$ & 89 & 144.66 & 3.6337 & 0.62394 & & & \\
\hline & $<5 \mathrm{yr}$ & 84 & 147.79 & 3.5976 & 0.76015 & & & \\
\hline & Total & 310 & & 3.6748 & 0.66250 & 5.461 & 3 & 0.141 \\
\hline \multirow{5}{*}{ ML } & $>1$ year & 67 & 199.40 & 3.8619 & 0.61435 & & & \\
\hline & 1 to $2 \mathrm{yr}$ & 70 & 164.75 & 3.4821 & 0.97554 & & & \\
\hline & 2 to $5 \mathrm{yr}$ & 89 & 134.94 & 3.3258 & 0.78462 & & & \\
\hline & $<5 \mathrm{yr}$ & 84 & 134.55 & 3.2262 & 0.96522 & & & \\
\hline & Total & 310 & & 3.4500 & 0.87965 & 26.422 & 3 & 0.000 \\
\hline \multirow{5}{*}{ CSR } & $>1$ year & 67 & 173.01 & 3.3910 & 0.60646 & & & \\
\hline & 1 to $2 \mathrm{yr}$ & 70 & 161.85 & 3.2800 & 0.73929 & & & \\
\hline & 2 to $5 \mathrm{yr}$ & 89 & 150.00 & 3.2652 & 0.62288 & & & \\
\hline & $<5 y r$ & 84 & 142.07 & 3.1786 & 0.68303 & & & \\
\hline & Total & 310 & & 3.2723 & 0.66474 & 5.189 & 3 & 0.158 \\
\hline \multirow{5}{*}{ PSA } & $>1$ year & 67 & 179.31 & 3.8985 & 0.47146 & & & \\
\hline & 1 to $2 y r$ & 70 & 160.27 & 3.7343 & 0.64040 & & & \\
\hline & 2 to $5 \mathrm{yr}$ & 89 & 147.94 & 3.7079 & 0.60307 & & & \\
\hline & $<5 \mathrm{yr}$ & 84 & 140.54 & 3.6286 & 0.62721 & & & \\
\hline & Total & 310 & & 3.7335 & 0.59781 & 8.054 & 3 & 0.045 \\
\hline \multirow{5}{*}{$\begin{array}{c}\text { Total service } \\
\text { quality } \\
\text { perception }\end{array}$} & $>1$ year & 67 & 188.34 & 3.7416 & 0.44156 & & & \\
\hline & 1 to $2 \mathrm{yr}$ & 70 & 166.79 & 3.5429 & 0.68392 & & & \\
\hline & 2 to $5 \mathrm{yr}$ & 89 & 140.43 & 3.4914 & 0.54644 & & & \\
\hline & $<5 \mathrm{yr}$ & 84 & 135.88 & 3.4173 & 0.63109 & & & \\
\hline & Total & 310 & & 3.5370 & 0.59303 & 16.669 & 3 & 0.001 \\
\hline
\end{tabular}

$\mathrm{CIU}=$ Cooperation among internal users. ML=Management leadership. $\mathrm{CSR}=$ Corporate social responsibility. PSA=Performance self-appraisal. Source: Prepared by the authors.

al $(p=0.005)$, compared to permanent and CAS workers, whose labor stability appear to have no impact on the factors studied. Similarly, employees who have fewer years with the institution obtained the highest levels regarding perception of management leadership $(p=0.00)$ and performance self-appraisal $(p=0.045)$, whereas the youngest employees ( 20 to 39 years old) obtained a higher level regarding perception of management leadership $(p=0.018)$ compared to older employees. These findings make us reflect on the improvement of the addressed subject: it is necessary to break down barriers and paradigms in response to the new social and global contexts that new generations encounter, where job stability and years of work at an institution do not guarantee that employees deliver a good service to internal customers, a fact that has an impact on the ultimate customer ("the public") satisfaction based on perception.
For that reason, it is necessary to implement strategies that enable the development of a culture based on service climate, where quality characterizes each employee (new and old), as climate strength moderates the relationship between employee perceptions and customer satisfaction (Schneider \& Nicole, 2002). Likewise, organizations that promote a culture based on service quality through employees that fulfill their functions, will offer not only a service, but a quality service, exceeding the expectations of the public (Simancas, 2012).

The findings of this study determined that internal user perception of service quality can be positive or negative. This ambivalence is attributed to the factors with a negative trend observed in management leadership and corporate social responsibility, which should be studied in order to achieve better performance standards in the management process and personal welfare. 


\section{CONCLUSIONS}

1. Public servants' perception of quality service in a public sector institution is ambivalent, with a favorable trend regarding perception of cooperation among internal users and performance self-appraisal factors.

2. In relation to contract modalities (permanent, CAS and outsourced), significant differences regarding perception of cooperation among internal users, management leadership and performance self-appraisal factors are observed, being the outsourced employees the group with higher level of positive perception compared to permanent and CAS employees.

3. The youngest employees (20 to 39 years old) present a higher level of perception regarding management leadership compared to the older employees.

4. In relation with the years of work at the institution, significant differences regarding perception of management leadership and performance self-appraisal factors are observed, being the employees with less than a year in the institution the group with a higher positive perception compared to those working longer.

\section{REFERENCES}

[1] Abedin, Z., Rahman, M. \& Mohiuddin, L. (2016). Service Quality Level and the Perception of Customers: Astudy on Nijhoom Tours $-5^{\star}$ Rated Travel and Tourism Company in Bangladesh. International Journal of Management Sciences and Business Research, 5(11), 109-118.

[2] Adler, A. (1973). Superiority and Social Interest: A Collection of Later Writings. New York, United States: Viking Press.

[3] Argyris, C. (1973). Personality and Organization Theory Revisited. Administrative Science Quarterly, 18(2), 141-167.

[4] Balmori, G. \& Flores, J. B. (2014). MECSI: Modelo para evaluar la calidad del servicio interno. Innovaciones de Negocios, 11(22), 191-213.

[5] Bellou, V. \& Andronikidis, A. (2008). The impact of internal service quality on customer service behaviour: Evidence from the banking sector. International Journal of Quality \& Reliability Management, 25(9), 943-954.
[6] Dean, A. M. (2004). Links between organisational and customer variables in services delivery: Evidence, contradictions and challenges. International Journal of Service Industry Management, 15(4), 332-350.

[7] Domínguez, H. (2006). El servicio invisible. Fundamento de un buen servicio al cliente. Colombia: ECOE Ediciones.

[8] Grönroos, C. (1984). A Service Quality Model and its Marketing Implications. European Journal of Marketing, 18(4), 36-44.

[9] Grönroos, C. (1985). Internal Marketing Theory and Practice. En T. M. Bloch, H. \& R. Block, G. D. Upah, et al. (eds.), Services Marketing in a Changing Environment (pp. 41-47). Chicago. United States: American Marketing Association.

[10] Grönroos, C. (1990). Relationship Approach to Marketing in Service Contexts: The Marketing and Organizational Behavior Interface. Journal of Bussines Research, (20), 3-11

[11] Guevara, U. L. de (2009). Medición de la satisfacción del cliente interno en una empresa de transformación. (Master Thesis). Universidad Veracruzana, Mexico.

[12] Gruca, T. S. \& Rego, L. (2005). Customer Satisfaction, Cash Flow, and Shareholder Value. Journal of Marketing, 69(3), 115-130.

[13] Hernández, R., Fernández, C. \& Baptista, P. (2014). Metodología de la investigación (6 ed.). Mexico D. F., Mexico: Mc Graw Hill Education.

[14] Kolb, D. A., Rubin, I. M. \& McIntyre, J. M. (1977). Psicología de las organizaciones. Experiencias. Madrid, Spain: Ediciones del Castillo.

[15] Lapassade, G. (1977). Grupos, organizaciones e instituciones. La transformación de la burocracia. Barcelona, Spain: Gedisa.

[16] Mayer, D. M., Ehrhart, M. G. \& Schneider, B. (2009). Service Attribute Boundary Conditions of the Service Climate-Customer Satisfaction Link. Academy of Management Journal, 52(5), 1034-1050.

[17] Miguel, J. M. de (1999). La organización como construcción social. Representación organizacional. (Doctoral Thesis). Universidad Autónoma de Madrid, Spain.

[18] Ospina, A. V. (2014). ¿Qué tan importante es el servicio al cliente interno y externo en una 
compañia? Retrieved from: https://repository. unimilitar.edu.co/bitstream/10654/13138/1/ ASTRID $\% 20$ VANESSA $\% 20$ OSPINA $\% 20$ MAHECHA.pdf.

[19] Paredes, M. T. (1997). La interdependencia empresarial: factor de calidad en la gestión Caso sector empresarial pesquero peruano. (Master Thesis). Universidad Nacional Mayor de San Marcos, Lima.

[20] Reardon, K. K. \& Enis, B. (1990). Establishing a Companywide Customer Orientation through Persuasive Internal Marketing. Management Communication Quarterly, 3(3), 376-387.

[21] Schneider, B. \& Nicole, A. N. (2002). Climate Strength: A New Direction for Climate
Research. Journal of Applied Psychology, 87(2), 220-229.

[22] Schneider, B., White, S. S. \& Paul, M. C. (1998). Linking Service Climate and Customer Perceptions of Service Quality: Test of a Causal Model. Journal of Applied Psychology, 83(2), 150-163.

[23] Simancas, R. (2012). Cliente interno y calidad de servicio en las organizaciones productivas. Dictamen Libre, (10-11), 81- 89.

[24] Zeithaml, V. A. (2000). Service Quality, Profitability, and the Economic Worth of Customer: What We Know and What We Need to Learn. Journal of the Academy of Marketing Science, 28(1), 67-85. 